\title{
Floral Scent in Wisteria: Chemical Composition, Emission Pattern, and Regulation
}

\author{
Yifan Jiang \\ College of Horticulture, Northwest Agricultural and Forestry University, Yangling, Shaanxi 712100, \\ China; and the Department of Plant Sciences, University of Tennessee, Knoxville, TN 37996
}

Xinlu Chen
Department of Plant Sciences, University of Tennessee, Knoxville, TN 37996

Hong Lin

Crop Diseases, Pests and Genetics Research Unit, USDA-ARS, 9611 South Riverbend Avenue, Parlier, CA 93648

Fei Wang ${ }^{1}$

College of Horticulture, Northwest Agricultural and Forestry University, Yangling, Shaanxi

712100, China

Feng Chen ${ }^{1}$

Department of Plant Sciences, University of Tennessee, 2431 Joe Johnson Drive, Knoxville, TN 37996

\begin{abstract}
Additional index words. ornamental plant, invasive species, terpenoids, methyl ester, headspace
Abstract. Volatile chemicals emitted from the flowers of chinese wisteria (Wisteria sinenesis) and japanese wisteria ( $W$. floribunda) were collected using a dynamic headspace technique and identified using gas chromatography-mass spectrometry; 28 and 22 compounds were detected from chinese wisteria and japanese wisteria flowers, respectively. These chemicals can be classified into four major classes, including fatty acid derivatives, benzenoids/phenylpropanoids, terpenoids, and nitrogen-containing compounds. Two monoterpenes, $(\mathrm{E})$ - $\beta$-ocimene and linalool, belonging to the class of terpenoids, were the most abundant compounds emitted from both species. Despite strong similarity, the floral volatile profiles of the two species displayed variations in both quality and quantity. Chinese wisteria was selected as a model for further study of volatile emission from different parts of flowers, emission dynamics, and regulation of floral scent production. Although floral volatiles were detected from all flower parts, petals emitted the most. The emission of floral volatiles displayed a diurnal pattern with the maximal emissions occurring during the daytime. This rhythmic pattern was determined to be light-dependent. Regulation of floral volatile emission by exogenous chemicals, including silver thiosulphate (an ethylene inhibitor), salicylic acid, and jasmonic acid, also was analyzed. Generally, jasmonic acid promoted the emission of floral volatiles. In contrast, neither silver thiosulphate nor salicylic acid showed a significant effect on floral volatile emission. The results presented in this article suggest that wisteria can serve as a useful system for exploring novel biochemistry of floral scent biosynthesis. They also build a foundation for the study of the biological/ecological significance of floral volatiles on the reproductive biology of wisteria species.
\end{abstract}

Wisteria, a genus of flowering plants in the Fabaceae family, includes 10 species of deciduous woody vines. Wisteria sinenesis and $W$. floribunda, two species native to East Asia, were introduced to North America in the 1800s. Because of their spectacular racemes in the spring, ease of establishment, hardiness, fast growth, and long lifespan, chinese wisteria and japanese wisteria have become two of the most popular flowering vines in the United States since their introduction. However, these same characters that have made chinese and japanese wisterias popular ornamental plants have also contributed to their aggressiveness. The vines of chinese and japanese wisterias easily overtake native shrubs and trees through shading and strangling.

Received for publication 12 May 2011. Accepted for publication 8 July 2011. This project was supported by the University of Tennessee Institute of Agriculture. Yifan Jiang is a recipient of a scholarship from the China Scholarship Council.

${ }^{1}$ Corresponding author. E-mail: fengc@utk.edu; xnwangfei521@126.com.
In the southeastern United States, chinese and japanese wisterias have been listed as invasive weeds (Trusty et al., 2007a). Although these plants can be controlled by manual removal or herbicides, their management in the wild can be very challenging.

To establish successful control program for these exotic wisterias, it will be important to understand the biology of these plants, especially their reproductive biology. Chinese and japanese wisterias are known for their fragrant flowers, a trait of important aesthetic value. The strong floral scent of wisterias may also be important for their reproduction. For many plants, floral scent plays a critical role in attracting pollinators for pollination (Wright and Schiestl, 2009). Wisteria flowers have been observed to be visited by bees, flies, and hummingbirds. Characterizing the volatile chemistry of wisteria flowers will be important for us to understand the role of floral scent of these species for their reproductive success, which may lead to new strategies for the control of these plants as exotic weeds. To this end, the first 
objective of this study was to identify the volatile compounds emitted from the flowers of chinese wisteria and japanese wisteria.

The composition and amounts of floral volatiles may change during anthesis. Such changes can be regulated by various internal and external factors, including light, flowering stages, diurnal pattern, and visitation by pollinators (Andrews et al., 2007). It is advantageous for the plants to have their scent output at maximal levels only when their potential pollinators are active so as to conserve energy (Dudareva and Pichersky, 2000). The rhythmic pattern of floral volatile emission has been demonstrated in many herbaceous species (Altenburger and Matile, 1988; Jiang et al., 2011; Lee et al., 2010). Its occurrence in woody species is much less studied. Therefore, the second objective of this study was to determine whether the emission of floral volatiles from wisteria displays a diurnal pattern and, if so, whether the diurnal pattern is regulated by light, circadian clock, or both.

The emission of plant volatiles may be regulated by plant hormones and signaling molecules. For example, ethylene, a hormone important for many aspects of plant biology, including flower development and senescence, has been shown to be involved in the regulation of floral volatile emissions in certain plant species (Jiang et al., 2011; Underwood et al., 2005). The jasmonic acid (JA) pathway was reported to regulate production and emission of volatiles from vegetative tissues for plant defense (Howe, 2004). In addition, salicylic acid (SA) also is involved in the production and release of some defense volatile compounds (Zhao et al., 2010). However, little is known about whether the JA and SA signaling pathways play a role in regulating the production and emission of floral volatiles, especially in woody plants. Thus, the third objective of this study was to determine whether silver thiosulphate [STS (an ethylene inhibitor)], JA, and SA can affect the emission of volatiles from wisteria flowers.

In this article, we report the comprehensive analysis of wisteria floral scent: chemical composition, emission patterns, and regulation. Volatile chemicals emitted from the flowers of chinese wisteria and japanese wisteria were collected using a dynamic headspace technique and identified using gas chromatography-mass spectrometry (GC-MS). Then chinese wisteria was used as a model for further analysis of emission dynamics and regulation of floral volatiles. The significance of floral scent to the reproductive biology of chinese and japanese wisterias is discussed.

\section{Materials and Methods}

Plant materials. Flower samples were collected from chinese wisteria and japanese wisteria plants grown on the campus of the University of Tennessee, Knoxville. Individual raceme with $\approx 30$ small flowers was detached from the plant in the morning, placed in a flask filled with distilled water, and transported to the laboratory for volatile analysis. Analyses were conducted between late April and early May 2010.

Volatile COLLECTION AND ANALYSIS. Volatiles emitted from flowers were collected in an open headspace sampling system (Analytical Research System, Gainesville, FL) under the controlled environment $\left[22^{\circ} \mathrm{C}\right.$ (day/night), $100 \mu \mathrm{E} \cdot \mathrm{m}^{-2} \cdot \mathrm{s}^{-1}$ illumination] as previously reported (Zhuang et al., 2008). A single raceme in a single flask with $\approx 150 \mathrm{~mL}$ of distilled water was placed in a glass chamber $(10 \mathrm{~cm}$ diameter and $30 \mathrm{~cm}$ tall) covered with a removable O-ring snap lid. Charcoal-purified air entered the chamber at a flow rate of $0.8 \mathrm{~L} \cdot \mathrm{min}^{-1}$ from the top through a Teflon hose. Volatiles were collected for $1 \mathrm{~h}$ through a SuperQ volatile collection trap (Analytical Research System) and eluted using $100 \mu \mathrm{L}$ methylene chloride containing 1-octanal $(0.003 \% \mathrm{w} / \mathrm{v})$ as an internal standard.

Floral volatiles were analyzed by a Shimadzu 17A gas chromatograph (17A; Shimadzu, Columbia, MD) coupled to a quadrupole mass selective detector (QP5050A; Shimadzu). Separation was performed on a column $30 \mathrm{~m} \times 0.25 \mathrm{~mm}$ i.d. $\times$ $0.25 \mu \mathrm{m}$ thick (Restek SHR5XLB; Shimadzu). Helium was the carrier gas (flow rate of $5 \mathrm{~mL} \cdot \mathrm{min}^{-1}$ ), a splitless injection (injection temperature $250{ }^{\circ} \mathrm{C}$ ) was used, and a temperature gradient of $6{ }^{\circ} \mathrm{C} \cdot \mathrm{min}^{-1}$ from $60{ }^{\circ} \mathrm{C}(3-\mathrm{min}$ hold $)$ to $300{ }^{\circ} \mathrm{C}$ was applied. Products were identified using the National Institute of Standards and Technology mass spectral database and by comparison of retention times and mass spectra with authentic reference compounds. Quantification was performed based on peak areas of mass chromatograms.

DETERMINATION OF EMISSON OF VOLATILES FROM DIFFERENT PARTS OF FLOWERS AND EMISSION DYNAMICS. Chinese wisteria was used to determine the emission of floral volatiles from different parts of flowers. Petals, sepals, stamens coupled with pistils, and pedicels were collected and analyzed for volatile emission under the controlled environment $\left[22^{\circ} \mathrm{C}\right.$ (day/night), $100 \mu \mathrm{E} \cdot \mathrm{m}^{-2} \cdot \mathrm{s}^{-1}$ illumination]. To determine the temporal emission dynamics of floral volatiles, inflorescences detached from chinese wisteria plants were subject to volatile collection under two light regimes: a normal light/dark (12/12 h) cycle and continuous darkness. Headspace collections were performed for $1 \mathrm{~h}$ followed by a 3-h interval for a total period of $48 \mathrm{~h}$.

The EFFECT OF SILVER THIOSUlPHATE, JASMONIC ACID, AND SALICYLIC ACID ON THE EMISSION OF THE FLORAL VOLATILES IN CHINESE WISTERIA. Detached inflorescences of chinese wisteria were placed in flasks filled with either distilled water (control) or solutions containing STS, SA, or JA. The concentrations of STS, SA, and JA were $0.2 \mathrm{~mm}, 5 \mathrm{~mm}$, and $10 \mu \mathrm{M}$, respectively, which are physiologically relevant based on previous studies (Chen et al., 2003; Sexton et al., 1995; Yang et al., 2006; Zhao et al., 2009). Headspace collections were performed from 4 to $5 \mathrm{~h}$ and from 24 to $25 \mathrm{~h}$ after the initiation of each treatment. SA, JA, and STS were purchased from Sigma-Aldrich (St. Louis, MO).

Hierarchical Clustering OF THE MAJOR COMPOUNDS. Emission rates of methyl esters from the flowers of chinese wisteria were used in a hierarchical cluster analysis, which was conducted using average linkage analysis in the Cluster 3.0 software (Version 1.39; Stanford University, Palo Alto, CA). Heat maps were created using the Java TreeView 1.60 software (Stanford University).

Statistical analysis. Analyses of volatile quantification in two species and with treatment of various solutions were conducted using SAS (Version 8.02; SAS Institute, Cary, NC) based on three biological replicates (volatiles collected from independent flower samples) and two technical replicates (repeat running of a same volatile sample on GC-MS) and SES were calculated for all mean values. Levels of significance were calculated using Student's $t$ test at $P \leq 0.05$.

\section{Results}

Characterization of Floral volatiles of Chinese and JANPANESE WISTERIAS. Both chinese and japanese wisterias emit a complex mixture of volatiles from their flowers: 28 volatiles 
were detected from chinese wisteria flowers and 22 from japanese wisteria flowers. Based on their structures and putative biosynthetic origin, these volatile compounds were classified into four major classes: fatty acid derivatives, benzenoids/ phenylpropanoids, terpenoids, and nitrogen-containing compounds (Table 1). Two monoterpenes, linalool and $(E)$ - $\beta$-ocimene, were predominant in floral volatiles for both species. Their emission rates were 11.2 and $4.0 \mu \mathrm{g} \cdot \mathrm{h}^{-1} \cdot \mathrm{g}^{-1}$ from chinese wisteria and
11.2 and $4.8 \mu \mathrm{g} \cdot \mathrm{h}^{-1} \cdot \mathrm{g}^{-1}$ from japanese wisteria, respectively. In contrast, the monoterpene 1,8-cineole was only detected in chinese wisteria and the monoterpene limonene was only detected in japanese wisteria. Sesquiterpenes including $\alpha$-copaene, (E)- $\beta$-caryophyllene, $\alpha$-humulene, germacrene $\mathrm{D}$, and elemol were exclusively detected from japanese wisteria, whereas (E)$\beta$-farnesene was detected from both chinese wisteria and japanese wisteria. Methyl benzoate was the most abundant benzenoid

Table 1. Floral volatiles and their emission rates from two wisteria species.

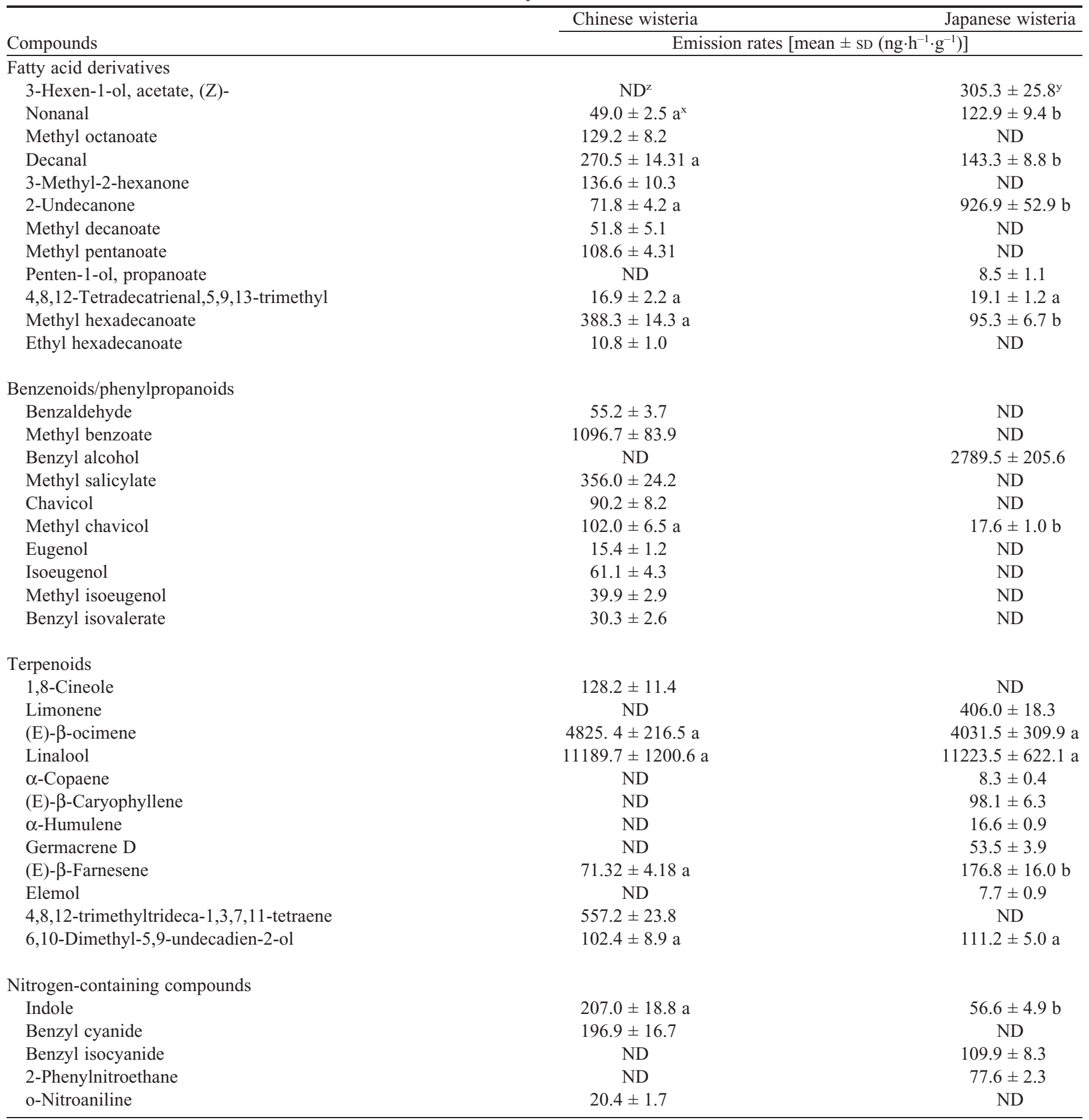

${ }^{\mathrm{z}}$ Not detected.

${ }^{\mathrm{y}}$ Means of three biological replicates and two technical replicates were presented.

$\mathrm{x}_{\mathrm{a}}$ and $\mathrm{b}$ represent significant difference between two species at $P \leq 0.05$ by Student's $t$ test. 
emitted from chinese wisteria $\left(1.1 \mu \mathrm{g} \cdot \mathrm{h}^{-1} \cdot \mathrm{g}^{-1}\right)$ flowers, whereas benzyl alcohol was the most abundant benzenoid in japanese wisteria $\left(2.8 \mu \mathrm{g} \cdot \mathrm{h}^{-1} \cdot \mathrm{g}^{-1}\right)$ flowers. Fatty acid derivatives account for $6.3 \%$ and $7.2 \%$ of total volatiles emitted from chinese and japanese wisterias, respectively. Chinese wisteria and japanese wisteria each emits three nitrogen-containing compounds from their flowers with indole being released from both plants (Table 1).

DETERMINATION OF EMISSION OF FLORAL VOLATILES FROM DIFFERENT PARTS OF CHINESE WISTERIA FLOWERS. Because chinese wisteria flowers emit more floral volatiles than japanese wisteria flowers both quantitatively and qualitatively (Table 1), chinese wisteria was selected as a model for further analysis of emission of floral volatiles from different parts of flowers, emission dynamics, and regulation of floral volatile emission. Volatiles were collected separately from petals, sepals, stamens and pistils, and pedicels. Twenty-seven, 15,11 , and 10 volatile compounds were identified from petals, sepals, stamens and pistils, and pedicels, respectively (Table 2 ). Petals showed the highest level of total emission rate $\left(15.5 \mu \mathrm{g} \cdot \mathrm{h}^{-1} \cdot \mathrm{g}^{-1}\right)$ among all four tissues. The total emission rate of pedicels was $\approx 10$ times lower than that of petals (Fig. 1). Although some volatiles showed tissue specificity, others including (E)- $\beta$-ocimene, linalool, nonanal, decanal, 2-undecanone, indole, 4,8,12-tetradecatrienal,5,9,13-trimethyl, methyl hexadecanoate, and ethyl hexadecanoate were detected from all parts of chinese wisteria flowers (Table 2).

EMISSION DYNAMICS OF FLORAL VOLATILES OF CHINESE WISTERIA. To examine the emission dynamics of floral volatiles, the volatiles emitted from the flowers of chinese wisteria were continuously checked (1-h collection followed by a 3 -h interval) for two photoperiods ( $12 / 12 \mathrm{~h}$ light/dark). The total emission rates of volatile compounds showed a diurnal pattern with the maximal emission occurrence during the daytime and the lowest level of emission at the night (Fig. 2A). The emission dynamics of three major compounds including linalool, methyl benzoate, and methyl hexadecanate, which are the most abundant volatiles from the chemical class of terpenoids, benzenoids, and fatty acid derivatives, respectively, were also analyzed. The emission patterns of these three compounds were similar to that of total volatile emission (Fig. 2B-D).

Table 2. Floral volatiles and their emission from different tissues of chinese wisteria flowers

\begin{tabular}{|c|c|c|c|c|}
\hline \multirow[b]{2}{*}{ Compounds } & Petal & Sepal & Stamen and pistil & Pedicel \\
\hline & \multicolumn{4}{|c|}{ Emission rates [mean $\left.\pm \mathrm{SD}\left(\mathrm{ng} \cdot \mathrm{h}^{-1} \cdot \mathrm{g}^{-1}\right)\right]$} \\
\hline \multicolumn{5}{|l|}{ Fatty acid derivatives } \\
\hline Nonanal & $88.2 \pm 6.4^{\mathrm{z}}$ & $63.7 \pm 8.3$ & $65.6 \pm 10.4$ & $67.21 \pm 7.1$ \\
\hline Decanal & $319.1 \pm 24.6$ & $244.1 \pm 37.1$ & $95.2 \pm 13.7$ & $86.5 \pm 8.1$ \\
\hline 3-Methyl-2-hexanone & $155.7 \pm 10.8$ & $210.5 \pm 26.0$ & ND & ND \\
\hline 2-Undecanone & $205.0 \pm 14.6$ & $67.5 \pm 5.2$ & $80.9 \pm 7.7$ & $51.5 \pm 4.3$ \\
\hline 4,8,12-Tetradecatrienal,5,9,13-trimethyl & $840.1 \pm 93.4$ & $140.8 \pm 18.1$ & $119.9 \pm 8.3$ & ND \\
\hline Methyl hexadecanoate & $1493.3 \pm 108.3$ & $444.5 \pm 52.7$ & $273.3 \pm 35.9$ & $242.4 \pm 28.9$ \\
\hline Ethyl hexadecanoate & $141.0 \pm 9.4$ & $34.7 \pm 4.4$ & $187.6 \pm 23.3$ & $14.2 \pm 1.3$ \\
\hline \multicolumn{5}{|l|}{ Benzenoids/phenylpropanoids } \\
\hline Methyl chavicol & $212.5 \pm 23.6$ & ND & ND & ND \\
\hline Eugenol & $70.8 \pm 9.5$ & ND & ND & ND \\
\hline Isoeugenol & $195.3 \pm 16.1$ & ND & ND & ND \\
\hline Methyl isoeugenol & $103.5 \pm 8.9$ & ND & ND & ND \\
\hline Benzyl isovalerate & $150.3 \pm 16.9$ & ND & ND & ND \\
\hline \multicolumn{5}{|l|}{ Terpenoids } \\
\hline 1,8-Cineole & ND & ND & $258.6 \pm 30.5$ & ND \\
\hline (E)- $\beta$-ocimene & $2255.9 \pm 142.1$ & $1464.9 \pm 182.8$ & $165.6 \pm 13.9$ & $75.5 \pm 9.7$ \\
\hline Linalool & $6392.4 \pm 502.5$ & $1358.2 \pm 105.5$ & $400.5 \pm 45.9$ & $366.2 \pm 41.2$ \\
\hline Farnesene & $139.3 \pm 17.7$ & ND & ND & ND \\
\hline
\end{tabular}

${ }^{\mathrm{z}}$ Means of three biological replicates and two technical replicates were presented.

${ }^{\mathrm{y}}$ Not detected. 


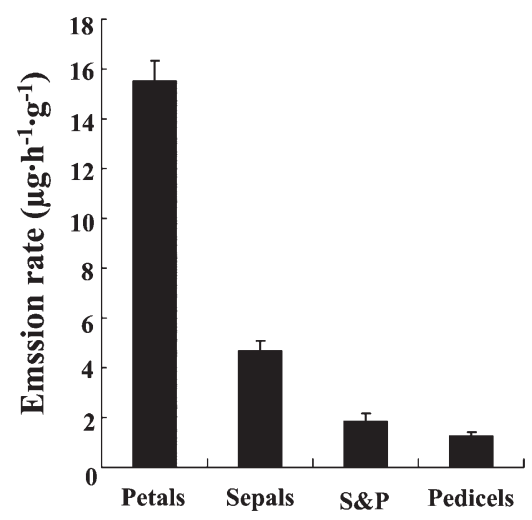

Fig. 1. Total emission rates of volatiles from four floral tissues of chinese wisteria, including petals, sepals, stamens and pistils (S\&P), and pedicels.

To determine the mechanism underlying the diurnal emission of floral volatiles of chinese wisteria, the flowers of chinese wisteria were placed under continuous darkness. The volatiles emitted from the flowers were continuously collected (1-h collection followed by a 3-h interval) for $48 \mathrm{~h}$. Under darkness, the emission of total floral volatiles lost the diurnal pattern and the levels of emission continued to decrease (Fig. 2A). Under continuous darkness, the three representative volatiles, linalool, methyl benzoate, and methyl hexadecaoic acid, displayed emission patterns very similar to that of total volatiles (Fig. 2A-D).

EFFECT OF SILVER THIOSUlPhATE, JASMONIC ACID, AND SALICYLIC ACID ON THE EMISSION OF THE FLORAL VOLATILES OF CHINESE WISTERIA. STS and SA did not show a significant effect on the total emission of chinese wisteria floral volatiles. In contrast, 24-h treatment by JA led to a significant increase in volatile emission (Fig. 3A). Interestingly, the three representative volatiles (linalool, methyl benzoate, and methyl hexadecanoate) responded differently to chemical treatment. The emission of linalool was unaffected by any of the chemicals at $4 \mathrm{~h}$ after the treatments. However, at $24 \mathrm{~h}$ after the treatment, JA significantly increased linalool emission (Fig. 3B). The emission of methyl benzoate was unaffected by STS and SA. In contrast, JA treatment increased the emission of methyl benzoate at both 4 and $24 \mathrm{~h}$ after the treatment (Fig. 3C). Interestingly, at $4 \mathrm{~h}$ after the treatment, JA reduced the emission of methyl hexadecanoate (Fig. 3D).

CluSTER ANALYSIS OF METHYL ESTERS FROM FLORAL VOLATILE EMISSION. One feature of the floral volatiles of chinese wisteria is the richness of methyl esters (Table 1). Multiple plant metabolites synthesized by the same enzyme or in a same biochemical pathway often exhibit a similar pattern of accumulation (Chen et al., 2009). Conversely, the compounds with a similar pattern of accumulation are likely the metabolites of a same biochemical pathway or even the products of a same enzyme. To gain insight into the biosynthesis of the methyl esters in chinese wisteria, the emission rates of eight methyl esters (methyl benzoate, methyl salicylate, methyl eugenol, methylchavicol, methyl octanoate, methyl pentanoate, methyl decanoate, and methyl hexadecanoate) under six conditions (control; chemical treatments with the STS, SA, and JA; and two different light regimes) were subject to cluster analysis. Based on the phylogeny and chemical structures, the eight compounds were grouped into three clades (Fig. 4). Methyl benzoate and methyl salicylate were grouped in clade I, whereas methyl eugenol
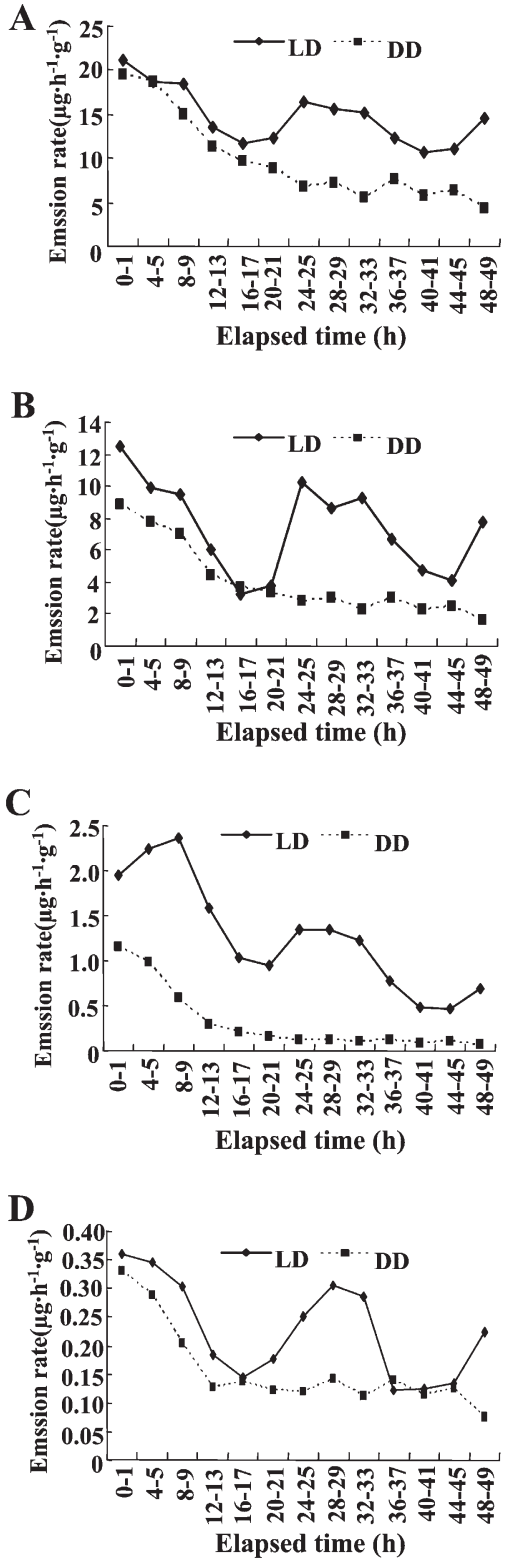

Fig. 2. Emission dynamics of total floral volatiles (A) and three individual major volatiles, including linalool (B), methyl benzoate $(\mathbf{C})$, and methyl hexadecanoate (D) from three different biosynthetic pathways, from chinese wisteria flowers under a normal light/dark cycle (LD) and continuous darkness (DD) during a 48-h period. Three replicates yielded similar results. Only emission rates from one representative analysis were presented.

and methylchavicol were grouped in clade II. Four fatty acid methyl esters, methyl octanoate, methyl pentanoate, methyl decanoate, and methyl hexadecanoate, were grouped together as clade III. Methyl benzoate and methyl salicylate are known to be synthesized by SABATH methyltransferases (Zhao et al., 2008). They can be the product of a same enzyme (Zhao et al., 2010). Methyl eugenol and methylchavicol are synthesized by the phenylpropene O-methyl transferases (Gang et al., 2002). The methyltransferases for the biosynthesis of fatty acid methyl esters have not been reported. However, they are very likely different from SABATH methyltransferases and phenylpropene O-methyl transferases as a result of the structural difference of substrates. Therefore, the cluster analysis suggests 
A

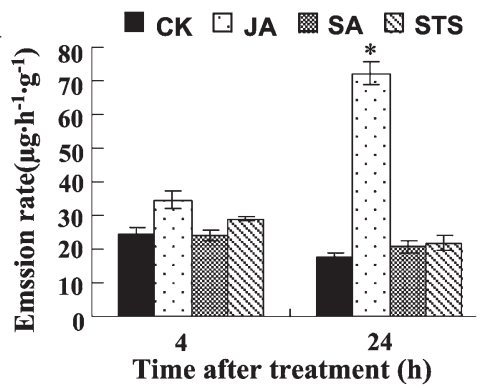

B

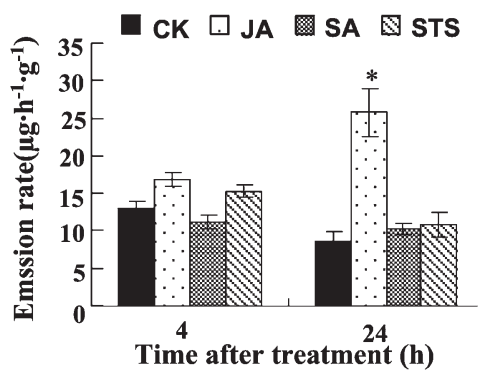

C

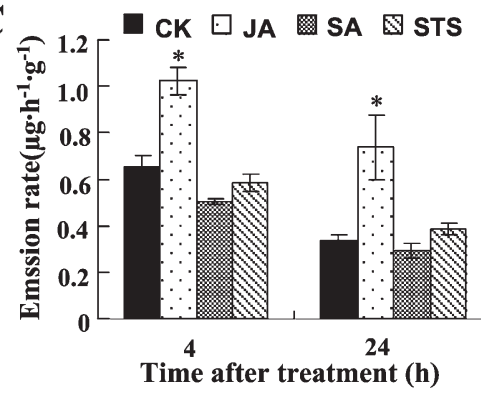

D

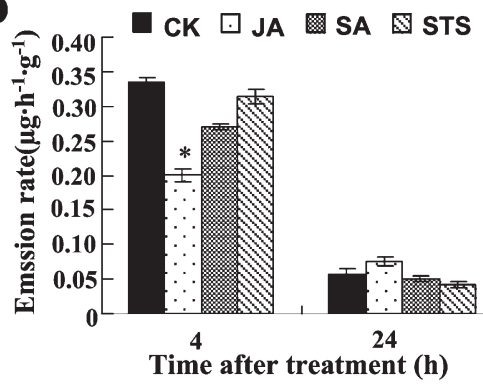

Fig. 3. Emission of total floral volatiles (A) and three individual major volatile compounds, including linalool (B), methyl benzoate (C), and methyl hexadecanoate (D) from untreated chinese wisteria flowers (CK) and chinese wisteria flowers treated by silver thiosulphate (STS), jasmonic acid (JA), and salicylic acid (SA). Volatiles were collected at two time points (4 h and $24 \mathrm{~h}$ ) after the beginning of the treatments; *statistical difference by Student's $t$ test at $P \leq 0.05$.

that three types of methyltransferase genes are involved in the biosynthesis of methyl esters from chinese wisteria flowers.

\section{Discussion}

In this article, we report the complete chemical profile of floral scents of two wisteria species. Flowers of both chinese wisteria and japanese wisteria emit a complex mixture of volatiles (Table 1). Similar to many other plants (Knudsen et al., 1993, 2006), the floral volatiles of the two species are dominated by compounds from four chemical classes: fatty acid derivative,

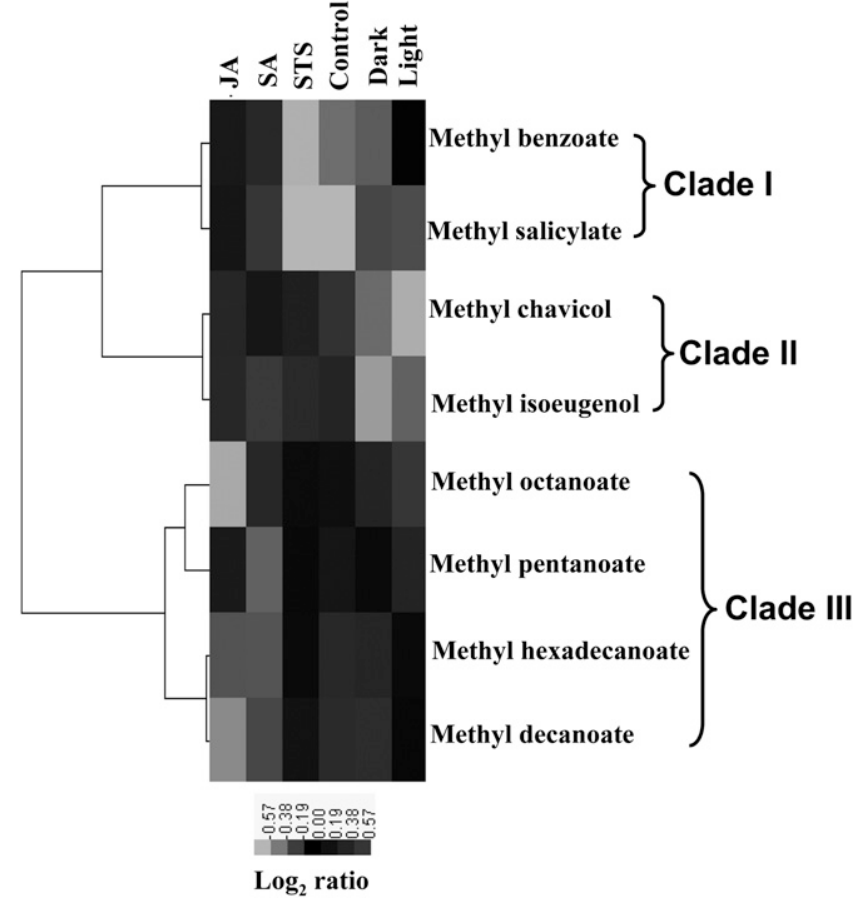

Fig. 4. Cluster analysis of eight methyl esters emitted from chinese wisteria flowers. Hierarchical K-means cluster analysis was performed based on emission rates of methyl esters from the flowers of chinese wisteria under six conditions described in this article that include control, salicylic acid treatment (SA), jasmonic acid treatment (JA), silver thiosulphate treatment (STS), a normal light/dark cycle (light), and continuous darkness (dark).

benzenoids/phenylpropanoids, terpenoids, and nitrogen-containing compounds. Linalool was the most abundant compound emitted from both species, accounting for approximately half of the total emission rate, which is consistent with the results of previous studies of wisteria floral volatiles using different methods (Joulain, 1987; Joulain and Tabacchi, 1994). Despite strong similarities, significant variations were observed. For example, chinese wisteria flowers emit only one sesquiterpene, whereas japanese wisteria flowers emit six sesquiterpenes (Table 1). In addition, although the number of benzenoids/phenylpropanoids from japanese wisteria flowers was much less than that from chinese wisteria flowers, benzyl alcohol was emitted from japanese wisteria at a high rate, but this compound was not detected from chinese wisteria flowers at all. These suggest that different biochemical pathways that lead to different floral chemistry may have evolved and may provide fitness benefits to the specific wisteria species.

Temporal emission patterns of floral volatiles may be regulated by both internal and external factors. In this study, we found that the emission of the floral volatiles from chinese wisteria exhibited a diurnal pattern (Fig. 2). The number of compounds and emission rates during the daytime were dramatically higher than those at night. Further analysis showed that the emission dynamics of total floral compounds and representative compounds (linalool, methyl benzoate, and methyl hexadecanoate) were light-dependent. We also showed in this study that different plant hormone and signaling molecules have different effects on emission of floral volatiles. STS, an ethylene inhibitor and SA, a defense signaling molecule, did not show a significant effect on the emission of volatiles, indicating that the emission of floral volatiles in chinese 
wisteria is independent of the ethylene and SA signaling pathways. In contrast, JA, another defense signaling molecule, affected the emission rates of linalool, methyl benzoate, and methyl hexadecanoate (Fig. 3B-D), suggesting that the JA signaling pathway plays an important role in regulating the biosynthesis and emission of floral volatiles in chinese wisteria.

The knowledge of floral chemistry of two wisteria species may kindle the interests of several research directions. One direction is to study the biosynthesis of wisteria floral volatiles, which may lead to novel biochemistry. Especially interesting is the molecular basis for the production of methyl esters, which are unusually rich in the floral volatiles of chinese wisteria. These metabolites include methyl octanoate, methyl decanoate, methyl pentanoate, methyl hexadecanoate, methyl salicylate, methyl benzoate, methyl eugenol, and methyl chavicol. Based on the known biochemistry, we can hypothesize that these metabolites are formed by the action of methyltransferases. Clustering analysis suggests that three types of methyltransferases may be involved in the production of methyl esters in chinese wisteria, which is supported by the current knowledge about plant methyltransferases. Methyl benzoate and methyl salicylate are synthesized by the action of carboxyl methyltransferase called benzoic acid/salicylic acid carboxyl methyltransferase that belongs to the SABATH family (Zhao et al., 2007, 2008, 2010). Methyl isoeugenol and methyl chavicol are formed by the action of O-methyltransferase, which belongs to a protein family different from the SABATH family (Gang et al., 2002). Fatty acid methyl esters may be formed by the action of a distinct family of methyltransferases yet to be identified. Therefore, chinese wisteria presents a useful model for studying the biosynthesis and regulation of the floral volatiles at the molecular and biochemical levels. With the advent of new technology in molecular biology and genomics such as next-generation sequencing, it will be relatively easy to identify and isolate responsible genes.

The second direction is to study the biological/ecological significance of floral volatiles on the reproductive biology of wisteria species. Because the flowers of wisterias are visited by various organisms, many of which can serve as pollinators, it is tempting to speculate that floral scent plays a critical role in the reproductive success of wisterias. Interestingly, a recent study showed that the majority of invasive wisteria plants in the southeastern United States analyzed are hybrids between chinese wisteria and japanese wisteria (Trusty et al., 2007b). New wisteria produced by interspecific or intraspecific hybridization gains more vigor to compete with native species. Therefore, hybridization may play an important role in the invasiveness of wisterias. The creation of a hybrid invasive wisteria species is likely promoted by pollinators guided by strong floral scent. In addition to their role in attracting pollinators, some compounds of floral volatiles may function as repellents against herbivores (Dudareva and Pichersky, 2000; Shrivastava et al., 2010). For example, benzyl cyanide and benzyl isocyanide, which were emitted from chinese wisteria and japanese wisteria, respectively, are toxic to many organisms including florivores. The knowledge of floral volatile chemistry of wisterias will help elucidate the roles of these compounds in pollination and defense. Manipulation of the key volatile compounds of wisteria species through breeding or genetic engineering may lead to novel wisteria ornamental plants that are less invasive.

\section{Literature Cited}

Altenburger, R. and P. Matile. 1988. Circadian rhythmicity of fragrance emission in flowers of Hoya carnosa R. Br. Planta 174:248-252.

Andrews, E.S., N. Theis, and L.S. Adler. 2007. Pollinator and herbivore attraction to Cucurbita floral volatiles. J. Chem. Ecol. 33:1682-1691.

Chen, F., H. Al-Ahmad, B. Joyce, N. Zhao, T.G. Kollner, J. Degenhardt, and C.N. Stewart. 2009. Within-plant distribution and emission of sesquiterpenes from Copaifera officinalis. Plant Physiol. Biochem. 47:1017-1023.

Chen, F., J.C. D’Auria, D. Tholl, J.R. Ross, J. Gershenzon, J.P. Noel, and E. Pichersky. 2003. An Arabidopsis thaliana gene for methylsalicylate biosynthesis, identified by a biochemical genomics approach, has a role in defense. Plant J. 36:577-588.

Dudareva, N. and E. Pichersky. 2000. Biochemical and molecular genetic aspects of floral scents. Plant Physiol. 122:627-633.

Gang, D.R., N. Lavid, C. Zubieta, F. Chen, T. Beuerle, E. Lewinsohn, J.P. Noel, and E. Pichersky. 2002. Characterization of phenylpropene O-methyltransferases from sweet basil: Facile change of substrate specificity and convergent evolution within a plant OMT family. Plant Cell 14:505-519.

Howe, G.A. 2004. Jasmonates as signals in the wound response. J. Plant Growth Regul. 23:223-237.

Jiang, Y., N. Zhao, F. Wang, and F. Chen. 2011. Emission and regulation of volatile chemicals from globe amaranth flowers. J. Amer. Soc. Hort. Sci. 136:16-22.

Joulain, D. 1987. The composition of the headspace from fragrant flowers: Further results. Flavour Fragrance J. 2:149-155.

Joulain, D. and R. Tabacchi. 1994. Two volatile $\beta$-chromenes from Wisteria sinensis flowers. Phytochem. 37:1769-1770.

Knudsen, J.T., R. Eriksson, J. Gershenzon, and B. Ståhl. 2006. Diversity and distribution of floral scent. Bot. Rev. 72:1-120.

Knudsen, J.T., L. Tollsten, and L.G. Bergstrom. 1993. Floral scents - A checklist of volatile compounds isolated by head-space techniques. Phytochemistry 33:253-280.

Lee, J., E. Sugawara, S. Yokoi, and Y. Takahata. 2010. Genotypic variation of volatile compounds from flowers of gentians. Breed. Sci. 60:9-17.

Sexton, R., A.E. Porter, S. Littlejohns, and S.C. Thain. 1995. Effects of diazocyclopentadiene (DACP) and silver thiosulfate (STS) on ethylene regulated abscission of sweet-pea flowers (Lathyrus odoratus L.). Ann. Bot. (Lond.) 75:337-342.

Shrivastava, G., M. Rogers, A. Wszelaki, D.R. Panthee, and F. Chen. 2010. Plant volatiles-based insect pest management in organic farming. Crit. Rev. Plant Sci. 29:123-133.

Trusty, L.J., B.G. Lockaby, W.C. Zipperer, and L.R. Goertzen. 2007a. Identity of naturalized exotic wisteria (Fabaceae) in the southeastern United States. Weed Res. 47:479-487.

Trusty, L.J., B.G. Lockaby, W.C. Zipperer, and L.R. Goertzen. 2007 b. Invasive wisteria in the southeastern United States: Genetic diversity, hybridization and the role of urban centers. Urban Ecosyst. 10:379395.

Underwood, B.A., D.M. Tieman, K. Shibuya, R.J. Dexter, H.M. Loucas, A.J. Simkin, C.A. Sims, E.A. Schmelz, H.J. Klee, and D.G. Clark. 2005. Ethylene-regulated floral volatile synthesis in Petunia corollas. Plant Physiol. 138:255-266.

Wright, G.A. and F.P. Schiestl. 2009. The evolution of floral scent: The influence of olfactory learning by insect pollinators on the honest signalling of floral rewards. Funct. Ecol. 23:841-851.

Yang, Y., J.S. Yuan, J. Ross, J.P. Noel, E. Pichersky, and F. Chen. 2006. An Arabidopsis thaliana methyltransferase capable of methylating farnesoic acid. Arch. Biochem. Biophys. 448:123-132.

Zhao, N., J.L. Ferrer, J. Ross, J. Guan, Y. Yang, E. Pichersky, J.P. Noel, and F. Chen. 2008. Structural, biochemical and phylogenetic analyses suggest that indole-3-acetic acid methyltransferase is an 
evolutionarily ancient member of the SABATH family. Plant Physiol. 146:455-467.

Zhao, N., J. Guan, J.L. Ferrer, N. Engle, M. Chern, P. Ronald, T.J. Tschaplinski, and F. Chen. 2010. Biosynthesis and emission of insect-induced methyl salicylate and methyl benzoate from rice. Plant Physiol. Biochem. 48:279-287.

Zhao, N., J. Guan, F. Forouhar, T.J. Tschaplinski, Z.M. Cheng, L. Tong, and F. Chen. 2009. Two poplar methyl salicylate esterases display comparable biochemical properties but divergent expression patterns. Phytochemistry 70:32-39.

Zhao, N., J. Guan, H. Lin, and F. Chen. 2007. Molecular cloning and biochemical characterization of indole-3-acetic acid methyl transferase from poplar. Phytochemistry 68:1537-1544.

Zhuang, X.F., W.E. Klingeman, J. Hu, and F. Chen. 2008. Emission of volatile chemicals from flowering dogwood (Cornus florida L.) flowers. J. Agr. Food Chem. 56:9570-9574. 